\title{
Analyst
}

Accepted Manuscript

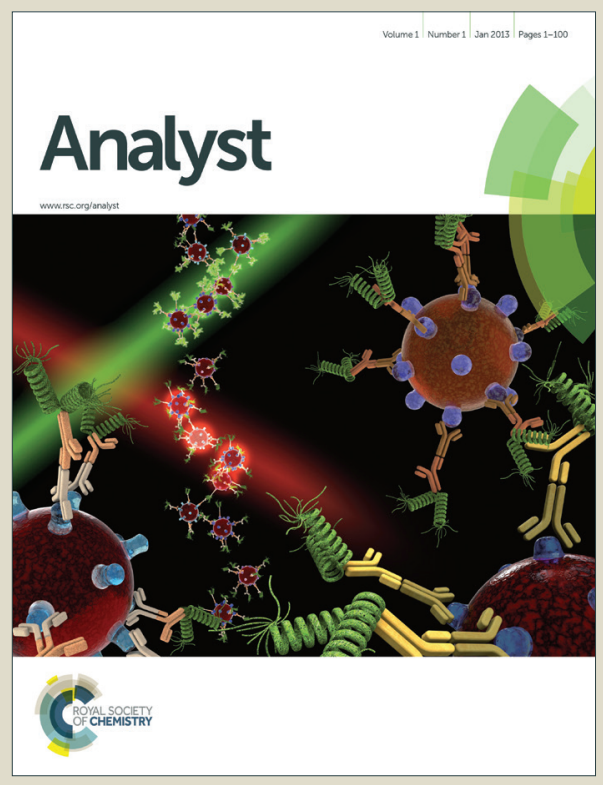

This is an Accepted Manuscript, which has been through the Royal Society of Chemistry peer review process and has been accepted for publication.

Accepted Manuscripts are published online shortly after acceptance, before technical editing, formatting and proof reading. Using this free service, authors can make their results available to the community, in citable form, before we publish the edited article. We will replace this Accepted Manuscript with the edited and formatted Advance Article as soon as it is available.

You can find more information about Accepted Manuscripts in the Information for Authors.

Please note that technical editing may introduce minor changes to the text and/or graphics, which may alter content. The journal's standard Terms \& Conditions and the Ethical guidelines still apply. In no event shall the Royal Society of Chemistry be held responsible for any errors or omissions in this Accepted Manuscript or any consequences arising from the use of any information it contains. 


\title{
Journal Name
}

\section{Using differential mobility spectrometry to measure ion solvation: An examination of the roles of solvents and ionic structures in separating quinoline-based drugs}

Received 00th January 20xx,

Accepted 00th January 20xx

Chang Liu, ${ }^{a}$ J. C. Yves Le Blanc, ${ }^{a}$ Jefry Shields, ${ }^{b}$ John S. Janiszewski, ${ }^{b}$ Christian leritano, ${ }^{c}$ Gene F. Ye, ${ }^{c}$ DOI: $10.1039 / x 0 \times x 00000 x$ Gillian F. Hawes, ${ }^{c}$ W. Scott Hopkins ${ }^{* c}$, and J. Larry Campbell ${ }^{* a}$

www.rsc.org/

\begin{abstract}
Understanding the mechanisms and energetics of ion solvation is critical in many scientific areas. Here, we present amethodlogy for studying ion solvation using differential mobility spectrometry (DMS) coupled to mass spectrometry. While in the DMS cell, ions experience electric fields established by a high frequency asymmetric waveform in the presence of a desired pressure of water vapor. By observing how a specific ion's behavior changes between the high- and low-field parts of the waveform, we gain knowledge about the aqueous microsolvation of that ion. In this study, we applied DMS to investigate the aqueous microsolvation of protonated quinoline-based drug candidates. Owing to their low binding energies with water, the clustering propensity of 8-substituted quinolinium ions was less than that of the 6- or 7substituted analogues. We attribute these differences to the steric hinderance presented by subtituents in the 8-position. In addition, these experimental DMS results were complemented by extensive computational studies that determined cluster structures and relative thermodynamic stabilities.
\end{abstract}

\section{Introduction}

Water has received more scientific attention than any other solvent due to its vital role in many biological and physicochemical processes. Accordingly, understanding the interaction of ions with water molecules in their immediate vicinity is of great interest in various areas, such as the nucleation of aerosols in atmosphere, ${ }^{1}$ drug solubility and lipophilicity, ${ }^{2}$ enzyme activity, ${ }^{3}$ and in the folding, nanofiltering, coagulation and flocculation process of proteins. ${ }^{4,} 5$ lon-water clusters have been extensively investigated in the liquid phase with spectroscopic methods, including infrared spectroscopy (IR), ${ }^{6,7}$ Raman spectroscopy, ${ }^{8}$ $X$-ray diffraction, ${ }^{9}$ and nuclear magnetic resonance (NMR) spectroscopy. ${ }^{10}$ In addition, information can be gained about the structure and reactivity of hydrated gas-phase ions using mass spectrometry (MS), ${ }^{11}$ which can provide a bridge between the chemistry of an isolated gas-phase ion and an ion present in bulk solution. Although still an area of debate, there have been studies showing the preservation of solution structure in the gas-phase. For example, several groups

\footnotetext{
a. SCIEX, 71 Four Valley Drive, Concord, ON, Canada. Email: larry.campbell@sciex.com.

b. Groton lab, Pfizer Global Research and Development, Groton, CT, USA

c. Department of Chemistry, University of Waterloo, Waterloo, ON, Canada. Email: scott.hopkins@uwaterloo.ca.

+ Footnotes relating to the title and/or authors should appear here. Electronic Supplementary Information (ESI) available: [details of any supplementary information available should be included here]. See DOI: $10.1039 / x 0 \times x 00000 x$
}

demonstrated that gas-phase hydrated peptides and proteins behaved similarly to those in the bulk solution, once a certain number of water molecules had clustered to the ion. ${ }^{5,12,13}$ In addition, the importance of aqueous microsolvation has been demonstrated when the solvation of protonated arginine by only a single water molecule was shown to promote the zwitterionic form as the most favourable gas-phase structure. ${ }^{14}$ In these experiments, solvated ions were generated external to the mass spectrometer using electrospray ionization (ESI) of aqueous solutions - a sometimes challenging and time-intensive process due to relatively low signal intensities. To improve upon this method, Rajabi and coworkers developed a hybrid Q-FTMS system, in which the solvation of bare (un-solvated) ions was decoupled from the ESI process; they controlled ion hydration by adjusting the water vapour pressure in the hexapole accumulation cell of their system. ${ }^{15}$ Subsequently, the temperature-dependent rate constants for ion desolvation could be obtained using blackbody infrared radiative dissociation (BIRD). ${ }^{16}$

Here, we present a study examining the microsolvation of gas-phase ions using differential mobility spectrometry (DMS). DMS is a technology that can separate gas-phase ions prior to analysis by MS. ${ }^{17-23}$ This technology has been used for both chemical noise elimination in LC-MS experiments, ${ }^{24}$ and isomeric ion separation, including stereoisomers, ${ }^{20,}$ 24-26 structural isomers, ${ }^{27-30}$ and even tautomers. ${ }^{31}$ In a DMS cell, an asymmetric separation voltage (SV) waveform that varies between high-field and low-field regimes is applied perpendicular to the ion transport flow. The different 
mobilities exhibited under the high- and low-electric fields results in ions acquiring a "zigzag" trajectory that can be angled toward one of the two parallel planar DMS electrodes (Figure S1). To steer ions back on-axis for successful sampling into the MS, a DC compensation voltage (CV) is required to restore the ion's trajectory. For a given SV, the magnitude of the $\mathrm{CV}$ appears to reflect the relative degree of microsolvation for various ions. ${ }^{32}$ Compared with other MS-based approaches for ion solvation investigations, the entire process of solvationdesolvation-resolvation can be studied with DMS-MS. In addition, the ion energy can be conveniently controlled with the magnitude of SV. We demonstrated this in a previous study, where a series of sterically hindered quaternary ammonium cations were used to probe ion/solvent clustering propensities within the DMS. ${ }^{32}$ Other DMS-based studies have also investigated the roles of ion/solvent clustering from different perspectives, ${ }^{33,34}$ including low percentage of gasphase water $(0.1-10,000 \mathrm{ppm})^{35}$.

Here, we turn our attention towards investigating the aqueous microsolvation of quinoline derivatives. Substituted quinolines are a group of molecules with fairly rigid planar structure that have been recognized as a class of medicinally privileged molecules. ${ }^{36}$ They exhibit a wide range of biological activities including antibacterial, ${ }^{37}$ anticancer, ${ }^{38}$ antiinflammatory, ${ }^{39}$ and anti-HIV. ${ }^{40}$ Therefore, it is of interest to find a fast, accurate method of assessing aqueous solubility of quinoline derivatives, since this may lead to enhanced rates of assaying the bioavailability for drug candidates. In this study, we examined the DMS behaviour of five pairs of substituted quinoline isomers (Figure 1) in a nitrogen environment seeded with water vapour. Specifically, we sought to investigate the correlation between the DMS clustering behaviour of protonated quinoline (quinolinium) derivatives, their structural differences, and their calculated water binding energies.

\section{Experimental}

\section{Chemicals}

Substituted quinolines listed in Figure 1 were purchased from ACES Pharma (Princeton, NJ) and were used without further purification. HPLC grade acetonitrile was bought from Caledon Laboratory Chemicals (Georgetown, ON, Canada), and formic acid was purchased from Sigma-Aldrich (Oakville, ON, Canada). Distilled deionized water (18M $\Omega$ ) was produced in-house using a Millipore (Billerica, MA, USA) Integral 10 water purification system.

\section{DMS-MS System}

A DMS system ${ }^{20}$ (SelexION $^{\mathrm{TM}}$, SCIEX, Concord, ON) was mounted in the atmospheric region between the 5500 QTRAP $^{{ }^{41}}{ }^{41}$ (SCIEX) system's sampling orifice and its electrospray ionization (ESI) source. The ESI probe was maintained at a voltage of $5500 \mathrm{~V}$. A constant gas flow in the DMS cell was achieved by the curtain gas flow $\left(\mathrm{N}_{2} ; 30\right.$ psi, 7.1 $\mathrm{L} / \mathrm{min}$ ) and the primary stage vacuum pumping of the MS system. The temperature of the transport gas in the DMS cell was maintained at $\sim 100{ }^{\circ} \mathrm{C}^{43}$ (DMS heater setting of $150^{\circ} \mathrm{C}$ ). Water was added to the curtain gas at $1.5 \%(\mathrm{v} / \mathrm{v})$ by a Perkin Elmer 200 liquid chromatograph pump (Waltham, MA). In each experiment, individual analytes were dissolved in solution (50/50 acetonitrile/water with $0.1 \%$ formic acid) to a concentration of $100 \mathrm{ng} / \mathrm{mL}$. Each solution was infused into the ESI source at a rate of $7 \mu \mathrm{L} / \mathrm{min}$.

The fundamental behavior of the $\mathrm{DMS}^{17-21,23,44}$ and the asymmetrical SV waveform ${ }^{23}$ are described elsewhere. For the experiments conducted in this study, the separation voltage (SV) was stepped from 0 to $4000 \mathrm{~V}$ (in 250-V increments). At each SV increment, the compensation voltage (CV) was scanned from $-60 \mathrm{~V}$ to $+15 \mathrm{~V}$ in $0.15-\mathrm{V}$ increments. During every CV step, multiple-reaction monitoring (MRM) signals for each analyte (Table S1) were recorded, yielding an ionogram. Each ionogram was fit with a Gaussian distribution and the average $\mathrm{CV}$ for the ion transmission was recorded. These data were plotted as dispersion plots, ${ }^{45}$ with the optimal CV for ion transmission as the $y$-axis and SV as the $x$-axis.

\section{Computational Methods}

To investigate the gas-phase microsolvation properties of the substituted quinolinium ions, it was necessary that an exhaustive search of ion-solvent cluster potential energy<smiles>Cc1ccc2nc(C)ccc2c1</smiles>

2,6-dimethylquinoline<smiles>Cc1ccc2cc(N)ccc2n1</smiles>

6-amino-2-methylquinoline<smiles>COc1ccc2nc(C)ccc2c1</smiles>

6-methoxy-2-methylquinoline<smiles>Cc1ccc2cc([N+](=O)[O-])ccc2n1</smiles>

2-methyl-6-nitroquinoline<smiles>Cc1ccc2ccc(Cl)cc2n1</smiles>

7-chloro-2-methylquinoline

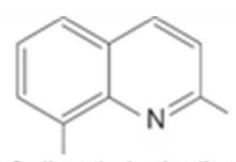

2,8-dimethylquinoline<smiles>Cc1ccc2cccc(N)c2n1</smiles>

8-amino-2-methylquinoline<smiles>COc1cccc2ccc(C)nc12</smiles>

8-methoxy-2-methylquinoline<smiles>Cc1ccc2cccc([N+](=O)[O-])c2n1</smiles>

2-methyl-8-nitroquinoline

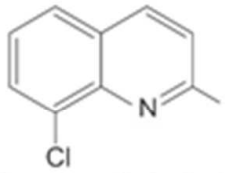

8-chloro-2-methylquinoline
Figure 1. The structures of the substituted quinolinnes. 
surfaces (PESs) be conducted. To this end, the basin-hopping (BH) algorithm was employed to map the low-energy regions of the PESs for quinolinium clusters that contain one water molecule. For two species, 2,6-dimethylquinolinium and 2,8dimethylquinolinum, clusters with up to 8 water molecules were investigated. Details of our implementation of the $\mathrm{BH}$ algorithm are reported elsewhere. ${ }^{32,46-48}$ Cluster systems were modeled using the AMBER force field. Partial charges for each monomer were calculated using the $\mathrm{CHelpG}$ partition scheme following geometry optimization at the B3LYP/6-311++G(d,p) level of theory. ${ }^{49,50}$ For each $\mathrm{BH}$ step, a random rotation of $20^{\circ}$ $\leq \theta \leq 20^{\circ}$ was applied about the body-fixed $x, y$, and $z$ axes for each water molecule. Solvent molecules were also randomly translated by $-0.7 \AA \leq \eta \leq 0.7 \AA$ in the $x, y$, and $z$ directions for each BH step. In the case of the amino-substituted species, a further random rotation of $-20^{\circ} \leq \phi \leq 20^{\circ}$ was applied to the amino group. BH searches explored $c a$. 10,000 structures for clusters containing one water molecule up to $c a$. 40,000 structures for clusters containing 8 water molecules. In all cases, protonation occurred on the ring nitrogen. Other protonation sites were investigated (e.g., protonation of the amine for the 6-and 8-amino derivatives), but were found to be significantly higher in energy.

Unique isomers identified by the $\mathrm{BH}$ routine were preoptimized at the HF/6-31G level of theory prior to geometry optimization at the $B 3 L Y P / 6-311++G(d, p)$ level of theory. Normal mode analysis was then conducted for each isomer to ensure that it corresponded to a local minimum. The global minima for 2,8-dimethylquinolinium $\bullet\left(\mathrm{H}_{2} \mathrm{O}\right)_{n}(n=1-8)$ are shown in Figure 2 and all other cluster structures and XYZ coordinates are available in the supporting information that accompanies this article. The global minimum for each cluster was then corrected for basis-set superposition error and empirical dispersion so as to calculate water binding energies accurately. ${ }^{51,52}$ Finally, to test the accuracy of DFT predictions, the electronic energies for the DFT-optimized global minima of 1-water clusters were improved by recalculation at the $\operatorname{CCSD}(T) / 6-311++G(d, p)$ level of theory. ${ }^{53}$
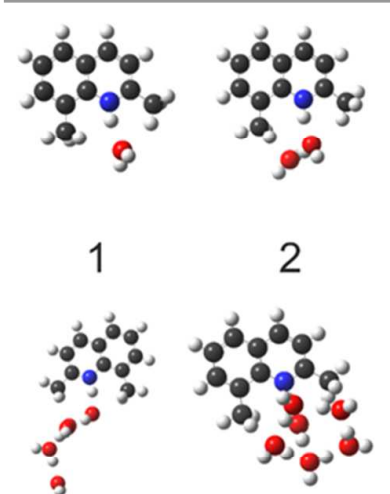

2

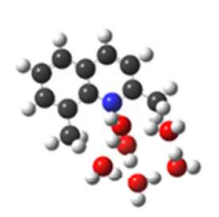

5
6

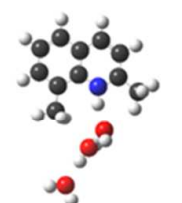

3

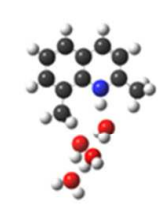

4

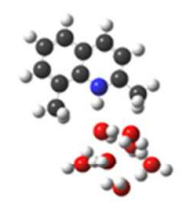

7

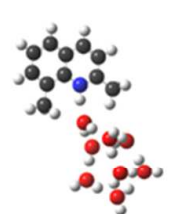

8
Figure 2. Global minimum structures for 2,8-dimethylquinolinium $\bullet\left(\mathrm{H}_{2} \mathrm{O}\right)_{n}(n=1$ $-8)$ as calculated at the B3LYP/6-311++G(d,p) level of theory.

\section{Results and discussion}

\section{How DMS reveals trends in an ion's solvation}

In DMS, a high-frequency, asymmetric RF waveform is applied perpendicular to the direction of ion transport. Ions can be separated on the basis of the difference in their mobility under high- and low- electric fields. Ion behaviour in the DMS cell has been explained by both the clustering model and the hardsphere scattering model, ${ }^{54}$ depending on the chemical modifier added into the ion transport gas flow. Three types of ion behaviour have been classified based upon the CVs needed for optimum ion transmission as a function of SV. ${ }^{19}$ Ions that exhibit Type A behaviour display negative $C V$ shifts with increasing SV. This behaviour can be rationalized by assuming a strongly clustering environment between ions and solvent molecules. With the presence of a polar chemical modifier, ions cluster with solvent molecules during the low-field portion of the asymmetric waveform and undergo declustering during the high-field half-cycle. Thus, under lowfield conditions ions exhibit a higher effective collision cross section, which impedes their mobility. lons that exhibit Type $C$ behaviour display positive CV shifts with increasing SV. This behaviour occurs as a consequence of field-induced ion acceleration, which manifests as higher ion collision probabilities at high SV. Thus, Type C ions exhibit lower relative mobility under high-field conditions, implying that lowfield clustering is negligible for Type $C$ ions (i.e., Type $C$ ions may be treated with a hard sphere model). ${ }^{40}$ Type $B$ behaviour results from a mixed mechanism where ion-solvent clustering dominates at low SV and hard sphere scattering dominates at high $S V .{ }^{55}$ Ions that exhibit Type B behaviour initially display negative CV shifts with increasing SV, but this trend reaches a minimum, eventually reversing such that positive CV shifts are observed at high SV. The CV minimum in a Type B ion dispersion plot (i.e., $\mathrm{CV}$ versus SV) can be viewed as a balance point for the Type A and Type C mechanisms. At SVs higher than the CV minimum, low-field clustering is increasingly inhibited by the energy imparted to the ions via collisions during the high-field half cycle. At SVs lower than the CV minimum, the smaller amplitude of the SV waveform results in reduced efficiency of ion de-clustering during the high-field half cycle. Consequently, the variation in effective collision cross section is not as great throughout the SV cycle and ions do not exhibit as extreme a differential mobility.

Subtle structural differences between quinolinium isomers greatly affect relative DMS behavior and microsolvation

The dispersion plots for the five substituted quinolinium isomer pairs are shown in Figure 3, and they reveal the differences that only minor structural variations can yield. The left column for Figure 3 shows the DMS behaviour of the quinolinium derivatives in a pure $\mathrm{N}_{2}$ environment (i.e., no chemical modifier), while the right column shows effect of adding $1.5 \% \mathrm{v} / \mathrm{v}$ water vapour to the $\mathrm{N}_{2}$ transport gas. In the absence of chemical modifiers, all quinolinium derivatives exhibit nearly identical Type $C$ behaviour. Two exceptions to this trend are the chloro- and nitro-substituted 2- 


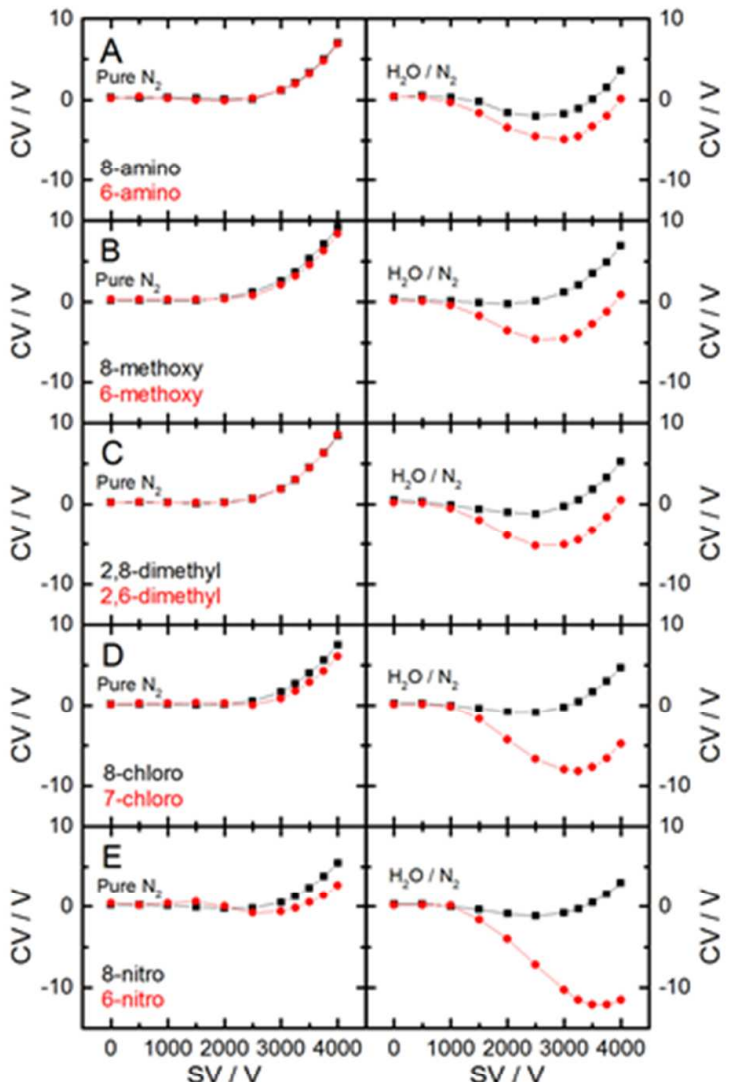

Figure 3 DMS dispersion plots for quinoline derivatives when (left) no modifier is added to the $\mathrm{N} 2$ transport gas and (right) when $1.5 \%$ water ( $\mathrm{v} / \mathrm{v}$ ) was added to the transport gas. (A) 6- and 8-amino-2-methylquinolinium,
(B) 6- and 8-methoxy-2-methylquinolinium, (C) 2,6- and 2,8dimethylquinolinium, (D) 7- and 8-chloro-2-methylquinolinium, and (E) 6and 8-nitro-2-methylquinolinium.

methylqunolinium isomers; while minimal, the separation of these isomers and the possible electronic effects played by their substituents, are the subject of an on-going study. However, upon the addition of water to the ion transport gas (Figure 3, right column), the majority of substituted quinolinium ions exhibit Type $\mathrm{B}$ behaviour. Note that the 8substituted-2-methylquinolinium ions also displayed a moderate reduction in transmitted CV values when water vapour was present, even if their overall DMS behavior could still be characterized as Type C. Overall, each different species/isomer displays a distinctly different dispersion plot. The observed differences in DMS behaviour in the presence of water vapour provide a qualitative view of clustering propensity for the substituted quinolinium ions. Species that cluster more strongly with water exhibit CV minima at higher SV than those that cluster weakly (Table 1). For example, consider the dispersion plots for the 6-nitro-2-methylquinoline and 8-nitro-2-methylquinolinium isomers (Figure 3E). The addition of water vapour to the $\mathrm{N}_{2}$ transport gas caused a significant change in the DMS behaviour of the 6-nitro derivative, but only a minor perturbation to the trajectory of the 8-nitro derivative. In other words, 6-nitro-2- methylquinolinium interacts much more strongly with the water vapour than 8-nitro-2-methylquinolinium.

\section{Relating DMS behaviors to ion-water binding energies using extensive computational modelling}

Based upon our initial energy optimization calculations, clustering between the quinolinium ions and water molecules was observed to occur in the vicinity of the protonated ring nitrogen atom (e.g., see Figure 2). The first water molecule binds directly to the charged $\mathrm{N}-\mathrm{H}$ moiety, with additional water molecules preferentially clustering amongst themselves via hydrogen-bonding typically forming 4- or 5-water cycles (for larger clusters). The water cluster structures are reminiscent of pure water clusters, ${ }^{56}$ but with slight distortions introduced by the 2-methylquinolinium derivatives. Distortion and disruption of the water cluster hydrogen bond network is particularly prominent for the case of 8-substituted 2methylquinolinium clusters owing to interaction with the substituents in the 2- and 8-positions (e.g., see Figure 2).

Upon identifying the global minimum for each cluster, solvent binding energies were calculated. While we report only zero point energy corrected values (i.e., $D_{0}$ ) here, we contend that these values represent the maximum binding energy for the methylquinolinium $\bullet\left(\mathrm{H}_{2} \mathrm{O}\right)_{n}$ species. This owes to the fact that the local temperature of the ions is unknown and dynamic (due to high-frequency field-induced heating). ${ }^{32}$ Table 1 provides the calculated binding energies for substituted methylquinolinium $\bullet\left(\mathrm{H}_{2} \mathrm{O}\right)_{1}$ clusters and reveals that, due to the similarity in the geometries of the various methylquinolinium derivatives, the calculated binding energies for the clusters all

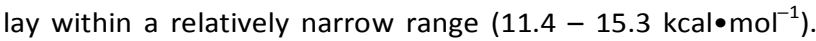
Note that the species with the lower binding energies among the isomeric pairs are all 8-substituted derivatives. Qualitatively, this accords well with the experimental observation that the 8-substituted species exhibit smaller magnitude SV minima than their 6- or 7-substituted isomers. A notable outlier to this trend is the 8-amino-2methylquinolinium $\bullet\left(\mathrm{H}_{2} \mathrm{O}\right)_{1}$ cluster, which is predicted to be one of the more strongly bound clusters

Table 1. SV at the DMS dispersion plot extrema for various methylquinolinium derivatives in an $\mathrm{N} 2$ environment seeded with $1.5 \% \mathrm{H} 2 \mathrm{O}(\mathrm{v} / \mathrm{v})$. Binding energies are calculated for a single water molecule at the $\operatorname{CCSD}(T) / 6-311++G(d, p)$ level of theory, and employ zero point energy corrections calculated at the B3LYP/6$311++G(d, p)$ level of theory.

\begin{tabular}{c|c|c}
\hline Analyte & SV (V) & Binding energy (kcal/mol) \\
\hline 8-amino-2-methylquinoline & 2360 & 15.2 \\
6-amino-2-methylquinoline & 2543 & 14.0 \\
8-methoxy-2-methylquinoline & 1519 & 11.8 \\
6-methoxy-2-methylquinoline & 2419 & 14.3 \\
2,8-dimethylquinoline & 1990 & 12.6 \\
2,6-dimethylquinoline & 2442 & 14.5 \\
8-chloro-2-methylquinoline & 1997 & 12.8 \\
7-chloro-2-methylquinoline & 2905 & 15.2 \\
2-methyl-8-nitroquinoline & 2358 & 11.4 \\
2-methyl-6-nitroquinoline & 3347 & 15.3 \\
\hline
\end{tabular}




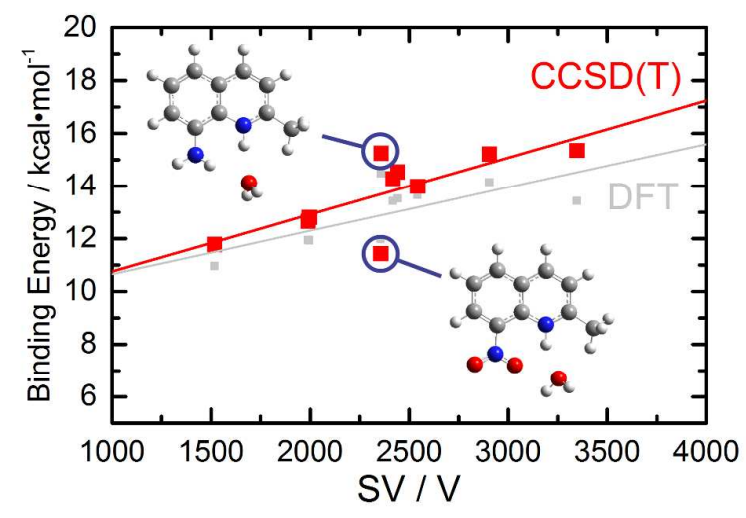

Figure 4. Calculated ion-water binding energies plotted as a function of the experimental $S V$ value at minimum $C V$. Red data points correspond to
binding energies calculated at the $\operatorname{CCSD}(T) / 6-311++G(d, p)$ level of theory employing zero point energy corrections calculated at the B3LYP/6$311++G(d, p)$ level of theory. Grey data points show the results of B3LYP/6$311++G(d, p)$ calculations that employed corrections for dispersion, basis set superposition error, and zero point energy. Solid lines show linear fits to the data sets.

$\left(B E=15.2 \mathrm{kcal} \bullet \mathrm{mol}^{-1}\right)$, but which exhibits relatively weak Type B DMS behavior. Closer inspection of the 8-amino-2methylquinolinium $\bullet\left(\mathrm{H}_{2} \mathrm{O}\right)_{1}$ cluster geometry shows that there is not one, but two $\mathrm{N}-\mathrm{H} \bullet \bullet \circ$ hydrogen bonds are formed upon complexation with the first water molecule. This binding motif - unique among the ions studied here - can potentially result in significant differences in high temperature thermodynamics and kinetics for the 8-amino species compared to the other methylquinolinium derivatives studied. The temperature-dependent behavior of all of the methylquinolinium species studied here is currently under investigation and will be the subject of a future publication. ${ }^{57}$

To quantify the observed clustering behaviour better, we have extracted the SV values at the CV minima for the 2methylquinolinium derivatives (see Table 1). This corresponds to the point of greatest dynamic clustering/de-clustering for these species within the water-seeded DMS cell. It is also implicitly related to the ion-solvent binding energies - species with greater water-binding energies are expected to exhibit $\mathrm{CV}$ minima that are shifted to higher SV. Consequently, one expects that a plot of the calculated binding energies versus these SV values should exhibit a linear trend, as is shown in Figure 4. Note that the water-clustering data have been corrected for the pure $\mathrm{N}_{2}$ background behaviour. Linear regression of the data set plotted in Figure 4 suggests that DMS can be used to measure substituted methylquinolinium $\bullet\left(\mathrm{H}_{2} \mathrm{O}\right)_{1}$ binding energies to within \pm 1.7 $\mathrm{kcal} \bullet \mathrm{mol}^{-1}$. However, much of the uncertainty in this fit can be attributed to two outlying data points; 8-amino-2methylquinolinium $\bullet \mathrm{H}_{2} \mathrm{O}$ and 2-methyl-8-nitroquinolinium (shown inset in Figure 4). Omitting these two data points from the fit reduces the uncertainty to $\pm 0.7 \mathrm{kcal} \bullet \mathrm{mol}^{-1}$. It is not yet clear why the 8-nitro derivative should also be an outlier from the observed clustering versus binding energy trend. Again, a detailed investigation of temperature dependence might prove enlightening.
We would like to stress that the observed correlation between the DMS behaviors with 1-water binding energies does not imply the absence of larger clusters during the dynamic clustering/de-clustering process. Indeed, the formation of larger clusters under low-field conditions is likely, ${ }^{58}$ but the extent to which ions cluster in the DMS environment (viz., the number of solvent molecules adsorbed) is an open question. DFT calculations for the 2,6-dimethyl and 2,8-dimethyl $(n=1-8)$ series (Figure S2) suggest that the most significant difference in water binding energies occurs for complexation with the first water molecule (up to $n=4$ ). ${ }^{32}$ Of course, this makes intuitive sense since the interaction between the first water molecule and the positively charged ring $\mathrm{N}-\mathrm{H}$ moiety is expected to be stronger than subsequent $\mathrm{H}$ bonding interactions between water molecules. Thus, isomeric substitutions that most greatly influence the initial complexation between the bare ion and the first water molecule should yield the largest variations in DMS behavior as this also affects the dynamics of subsequent cluster growth.

\section{Conclusions}

In this study, we used substituted quinolinium ions as models to demonstrate the ability of DMS to probe the relative strength of ion microsolvation. With the addition of water in the ion transport gas, isomeric species were distinguishable: a lower $\mathrm{CV}$ was required for the transmission of 8-substituted-2methylquinolinium ions through the DMS cell, because of their weaker ion solvation strength compared to their 6- or 7substituted isomers. These results were corroborated by extensive basin hopping PES searches and electronic structure calculations to determine the binding energies of the ion/solvent clusters.

\section{Acknowledgements}

We gratefully acknowledge high performance computing support from the SHARCNET consortium of Compute Canada. We are also grateful to Professor Terry McMahon (University of Waterloo) and Drs. Bradley Schneider and Tom Covey (AB SCIEX) for helpful conversations. We thank the Natural Sciences and Engineering Research Council of Canada (NSERC) for financial support through the ENGAGE grant (EGP \#44935413) and ENGAGE Plus grant (EGP \#463974-14).

\section{Notes and references}

1. R. Zhang, A. Khalizov, L. Wang, M. Hu and W. Xu, Chem. Rev., 2012, 112, 1957-2011.

2. C. A. Lipinski, F. Lombardo, B. W. Dominy and P. J. Feeney, Adv. Drug Deliv. Rev., 1997, 23, 3-25.

3. M. Tait and F. Franks, Nature, 1971, 230, 91-94.

4. B. Bagchi, Chem. Rev., 2005, 105, 3197-3219.

5. M. F. Jarrold, Ann. Rev. Phys. Chem., 2000, 51, 179-207.

6. D. E. Moilanen, D. Wong, D. E. Rosenfeld, E. E. Fenn and

M. Fayer, Proc. Natl. Acad. Sci., 2009, 106, 375-380.

7. Y. Rezus and H. Bakker, Phys. Rev. Lett., 2007, 99, 148301. 
8. I. A. Heisler, K. Mazur and S. R. Meech, J. Phys. Chem. B, 37. 2011, 115, 1863-1873.

9. T. Imoto, L. N. Johnson, A. C. T. North, D. C. Phillips and J. 38. A. Rupley, Enzymes, 1972, 7, 668-868.

10. G. Engel and H. Hertz, Berichte der Bunsengesellschaft für physikalische Chemie, 1968, 72, 808-834.

11. C. Desfrancois, S. Carles and J. Schermann, Chem. Rev. 2000, 100, 3943-3962.

12. S. E. Rodriguez-Cruz, J. S. Klassen and E. R. Williams, J. Am. Soc. Mass Spectrom., 1999, 10, 958-968.

13. S.-W. Lee, P. Freivogel, T. Schindler and J. Beauchamp, J. Am. Chem. Soc., 1998, 120, 11758-11765.

14. M. R. Bush, J. S. Prell, R. J. Saykally and E. R. Williams, J. Am. Chem. Soc., 2007, 129, 13544-13553.

15. K. Rajabi, M. L. Easterling and T. D. Fridgen, J. Am. Soc. Mass Spectrom., 2009, 20, 411-418.

16. R. C. Dunbar, Mass Spectrom. Rev., 2004, 23, 127-158.

17. G. Eiceman and Z. Karpas, Ion Mobility Spectrometry, CRC Press, Boca Raton, Florida, 2nd edn., 2005.

18. E. V. Krylov, E. G. Nazarov and R. A. Miller, Int. J. Mass Spectrom., 2007, 266, 76-85.

19. R. W. Purves and R. Guevremont, Anal. Chem., 1999, 71, 2346-2357.

$20 . \quad$ B. B. Schneider, T. R. Covey, S. L. Coy, E. V. Krylov and E. G. Nazarov, Int. J. Mass Spectrom., 2010, 298, 45-54.

21. A. A. Shvartsburg, Differential Ion Mobility Spectrometry: Nonlinear lon Transport and Fundamentals of FAIMS, CRC Press, Boca Raton, Florida, 2009.

22. J. L. Campbell, J. C. Y. Le Blanc and R. G. Kibbey, Bioanalysis, 2015, 7, 853-856.

23. B. B. Schneider, E. G. Nazarov, F. Londry, P. Vouros and T. R. Covey, Mass Spectrom. Rev., 2015, in press.

24. W. Jin, M. Jarvis, M. Star-Weinstock and M. Altemus, Anal. Bioanal. Chem., 2013, 405, 9497-9508.

25. R. W. Purves, D. A. Barnett and R. Guevremont, Int. J. Mass Spectrom., 2000, 197, 163-177.

26. E. W. Robinson and E. R. Williams, J. Am. Soc. Mass Spectrom., 2005, 16, 1427-1437.

27. D. A. Barnett, B. Ells, R. Guevremont and R. W. Purves, J. Am. Soc. Mass Spectrom., 1999, 10, 1279-1284.

28. W. B. Parson, B. B. Schneider, V. Kertesz, J. J. Corr, T. R. Covey and G. J. Van Berkel, Rapid Commum. Mass Spectrom., 2011, 25, 3382-3386.

29. M. R. Noestheden, J. V. Headley, K. M. Peru, M. P. Barrow, L. L. Burton, T. Sakuma, P. Winkler and J. L. Campbell, Environ. Sci. Technol., 2014, 48, 10264-10272.

30. A. T. Maccarone, J. Duldig, T. W. Mitchell, S. J. Blanksby, E. Duchoslav and J. L. Campbell, Journal of lipid research, 2014, 55, 1668-1677.

31. J. L. Campbell, J. C. Y. Le Blanc and B. B. Schneider, Anal. Chem., 2012, 84, 7857-7864.

32. J. L. Campbell, M. Zhu and W. S. Hopkins, J. Am. Soc. Mass Spectrom., 2014, 25, 1583-1591.

33. D. Auerbach, J. Aspenleiter and D. A. Volmer, J. Am. Soc. Mass Spectrom., 2014, 25, 1610-1621.

34. A. Kafle, S. L. Coy, B. M. Wong, A. J. Fornace, J. J. Glick and P. Vouros, J. Am. Soc. Mass Spectrom., 2014, 25, 10981113.

35. N. Krylova, E. Krylov, G. Eiceman and J. Stone, The Journal of Physical Chemistry A, 2003, 107, 3648-3654.

36. Beena and D. S. Rawat, Med. Res. Rev., 2013, 33, 693-764.
A. Mahamoud, J. Chevalier, A. Davin-Regli, J. Barbe and J. M. Pages, Curr. Drug Targets, 2006, 7, 843-847.

B. Heiniger, G. Gakhar, K. Prasain, D. H. Hua and T. A. Nguyen, Anticancer Res., 2010, 30, 3927-3932.

P. Leatham, H. Bird, V. Wright, D. Seymour and A. Gordon, Eur. J. Rheumatol. Infl., 1983, 6, 209.

L. Strekowski, J. L. Mokrosz, V. A. Honkan, A. Czarny, M. T. Cegla, R. L. Wydra, S. E. Patterson and R. F. Schinazi, J. Med. Chem., 1991, 34, 1739-1746.

41. B. A. Collings and M. A. Romaschin, J. Am. Soc. Mass Spectrom., 2009, 20, 1714-1717.

42. M. Guna and T. A. Biesenthal, J. Am. Soc. Mass Spectrom. 2009, 20, 1132-1140.

43. B. B. Schneider, T. R. Covey, S. L. Coy, E. V. Krylov and E. G. Nazarov, Anal. Chem., 2010, 82, 1867-1880.

44. E. V. Krylov, S. L. Coy, J. Vandermey, B. B. Schneider, T. R. Covey and E. G. Nazarov, Rev. Sci. Instrum., 2010, 81, 024101.

45. D. S. Levin, R. A. Miller, E. G. Nazarov and P. Vouros, Anal. Chem., 2006, 78, 5443-5452.

46. D. J. Wales and J. P. Doye, J. Phys. Chem. A, 1997, 101 5111-5116.

47. W. S. Hopkins, R. A. Marta and T. B. McMahon, J. Phys. Chem. A, 2013, 117, 10714-10718.

48. M. J. Lecours, W. T. Chow and W. S. Hopkins, J. Phys. Chem. A, 2014, 118, 4278-4287.

49. A. D. Becke, J. Chem. Phys., 1993, 98, 5648-5652.

50. C. T. Lee, W. T. Yang and R. G. Parr, Phys. Rev. B, 1988, 37, 785-789.

51. S. Grimme, J. Antony, S. Ehrlich and H. Krieg, J. Chem. Phys., 2010, 132, 154104.

52. S. Simon, M. Duran and J. Dannenberg, J. Chem. Phys., 1996, 105, 11024-11031.

53. K. Raghavachari, G. W. Trucks, J. A. Pople and M. HeadGordon, Chem. Phys. Lett., 1989, 157, 479-483.

54. E. V. Krylov and E. G. Nazarov, Int. J. Mass Spectrom. 2009, 285, 149-156.

55. W. S. Hopkins, Mol. Phys., 2015, in press.

56. D. J. Wales and M. P. Hodges, Chem. Phys. Lett., 1998, 286, 65-72.

57. C. Liu, J. C. Y. Le Blanc, N. Faruk, J. Shields, J. S. Janiszewski, I. C., G. F. Ye, G. F. Hawes, P. N. Roy, W. S. Hopkins and J. L. Campbell, manuscript in preparation, 2015.

58. J. L. Campbell, A. M.-C. Yang, L. R. Melo and W. S. Hopkins, Submitted, 2015. 